\title{
In Silico Safety Pharmacology on Intersubject Variability Population of Models: A Regression Model Approach
}

\author{
AS de la Nava ${ }^{1,2}$, A Liberos ${ }^{1}$, I Hernandez-Romero ${ }^{1}$, MS Guillem $^{3}$, F Atienza ${ }^{1}$, F Fernandez-Aviles $^{1}$, \\ AM Climent ${ }^{1}$ \\ ${ }^{1}$ Hospital GU Gregorio Marañón, IiSGM, CIBERCV, Madrid, Spain \\ ${ }^{2}$ Universidad Carlos III de Madrid, Spain \\ ${ }^{3}$ ITACA, Universitat Politècnica de València, Spain
}

\begin{abstract}
Safety pharmacology aims at detecting undesirable effect of drugs during its development. However, limitations are present at both in-vitro and in-silico level because of its low detection efficacy during this process.

In this work, the effect of drugs at tissue level was studied and inducibility in a multivariable scenario including 127 models tested for two different tissue sizes (basal and dilated) and two conditions (no drug and isoproterenol) was obtained.

From these models, maintenance duration (MD) of the reentry was calculated and a regression model based on Canonical Correlation Analysis (CCA) was implemented to evaluate the proarrhythmic effect of isoproterenol depending on model size.

The number of models with AF maintenance was larger for dilated atria and isoproterenol. CCA analysis obtained 96\% accuracy on an arrhythmogenicity test set for basal size and $100 \%$ on the dilated one.

A new promising methodology was proposed for safety pharmacology including variability between patients, setting the base for personalized medicine.
\end{abstract}

\section{Introduction}

The aim of safety pharmacology is to detect potential undesirable pharmacodynamic effects of new chemical entities during drug development process. Cardiotoxicity is one of the main side effects of most new chemical entities. Unfortunately, the cardiotoxic side effects of many new chemical entities are only detected during phase I and phase II clinical trials, whereas in-vitro and preclinical studies did not detect any malignant effect.

Limitations of in-vitro and preclinical trials are in part due to the lack of variability of those research models. Humans present important inter-subject variabilities in both their anatomical (i.e. size) and electrophysiological properties. Populations of mathematical models have appeared as a useful tool to evaluate drug safety in-silico accounting for the variability between patients [1]. However, standard mathematical models at tissue level require time-consuming simulations and complex inducibility protocols to confirm or discard re-entries and arrhythmia maintenance.

In this paper we present a novel method to evaluate safety pharmacology in-silico. This novel method allows to estimate the pro-arrhythmic or anti-arrhythmic effect of a drug on each individual member of population of models without the need of performing time-consuming simulations.

Specifically, a proof of concept study is presented evaluating the effect of isoproterenol (a non-selective $\beta$ adrenoreceptor agonist) on atrial fibrillation (AF) inducibility in a multivariable scenario, including a population of mathematical models with both variability on ion conductances and on tissue size. This novel approach will be validated by comparing the prediction of the novel methodology with the results of 2D mathematical model simulations.

\section{Materials and Methods}

\subsection{Population of models}

An experimentally population of 127 human AF tissue mathematical models [2] reproducing the variability observed in 149 chronic AF patients was used [1]. As described before [2], a atrial cardiomyocytes electrophysiological model was used as basis for the generation of a population of models in which $\mathrm{g}_{\mathrm{Na}}, \mathrm{I}_{\mathrm{Nak}}, \mathrm{g}_{\mathrm{K} 1}$, $\mathrm{g}_{\mathrm{CaL}}, \mathrm{g}_{\mathrm{Kur}}, \mathrm{I}_{\mathrm{KCa}}, \mathrm{K}_{\mathrm{o}}, \mathrm{Na}_{0}$ and the diffusion was varied.

Substrate size is an anatomical feature that determines the maintenance of AF [3]. For this purpose, two different square planes, with $100 \mu \mathrm{m}$ inter-nodal distance, corresponding to normal $\left(400 \times 400\right.$ nodes, $\left.15 \mathrm{~cm}^{2}\right)$ and a dilated $\left(450 \mathrm{x} 450\right.$ nodes, $\left.20 \mathrm{~cm}^{2}\right)$ atria were tested in order to evaluate the dependence on tissue size on arrhythmogeneity. 


\subsection{Safety pharmacology}

To simulate the effect of a drug, a simple pore model was implemented in the model:

$$
G_{i}=G_{0} \cdot \frac{1}{1+\frac{\left[D_{i}\right]}{I C_{50}}}
$$

Where $\mathrm{G}_{0}$ is the initial conductance of the channel, $\mathrm{D}_{\mathrm{i}}$ is the concentration of the drug and $\mathrm{IC}_{50}$ is the concentration of the drug that reduces by $50 \%$ the channel current. For this experiment, the simulated drug was isoproterenol. Isoproterenol is a a non-selective $\beta$ adrenoreceptor agonist used for the treatment of bradycardia (slow heart rate) but that can present pro-arrhythmic effects and AF inducibility in some patients [4]. Specifically, $\mathrm{I}_{\mathrm{Na}}, \mathrm{I}_{\mathrm{Kr}}$ and $\mathrm{I}_{\mathrm{CaL}}$ were modified and the concentration for the drug was set to $5 \cdot 10$ ${ }^{3} \mathrm{mM}[5]$.

\subsection{Workflow of the model}

For each of the 127 mathematical models and tissue sizes, reentrant activity was induced with the S1S2 protocol. Optimal S2 triggering was computed for each model based on its specific properties and on the sodium channel availability. Furthermore, a minimum voltage for triggering was also added as a boundary condition. The threshold established for both sodium channel availability and voltage were $4 \cdot 10^{-5}$ u.a. and $-75 \mathrm{mV}$ respectively.

Following an S1S2 protocol, simulation continued for 5 seconds. At that point, if the reentrant activity continued, the drug was applied to evaluate its antiarrhythmic effect and the simulation was continued for 5 more seconds. In case the reentrant activity did not continue, the drug was applied to the model and S1S2 protocol was applied again to evaluate the potential proarrhythmic effect of the drug.

For each of the four scenarios (i.e. (A) basal $15 \mathrm{~cm}^{2}$, (B) drug $15 \mathrm{~cm}^{2},(\mathrm{C})$ basal $20 \mathrm{~cm}^{2}$, (D) drug $20 \mathrm{~cm}^{2}$ ) the duration of the reentrant activity after the induction protocol was quantified. Effects of the drug on AF inducibility were defined as the maintenance difference (MD), or the difference in time between AF duration with and without drug infusion (groups A vs. B and C vs. D) or for different atrial sizes (A vs. C).

Then, after computing the effect of the drug or the dependence with size, each simulation was classified as: (1) antiarrhythmic if MD was decreased, (2) proarrhythmic if MD was increased or (3) no effect if MD remained unchanged.

All simulations were performed in a GPU nVIDIA TESLA C2057 system [1].

\subsection{Canonical Correlation Analysis}

The goal of the novel methodology was to predict the proarrhythmic, antiarrhythmic or neutral effect of the drug on each mathematical model without the need of performing the long simulations described in section 2.3. Specifically, a regression model dependent on conductances and tissue size was developed. The regression model was estimated by using Canonical Correlation Analysis (CCA), a dimension reduction method that maximizes the correlations between two multidimensional variables in a compact subspace.

CCA was independently trained for over $80 \%$ randomly selected models of the two population of models (i.e. 101 models in a basal atrium and 101 models in the dilated atria) to confirm the robustness of the methodology. CCA transformation matrices were used as a regression method to estimate the predictability of the method over the test set (i.e. 26 mathematical models for each of the two sizes).

\section{Results}

\subsection{Effect of size on arrhythmogenicity}

In Figure 1 the inducibility protocol for 1 of the 127 models is depicted, both for basal tissue (i.e. $15 \mathrm{~cm}^{2}$ ) and for dilated tissue (i.e. $20 \mathrm{~cm}^{2}$ ). Notice that for both atrial sizes, the S2 interval captured and induced reentries. Nevertheless, in the smaller atria the arrhythmia selfterminate after 3 reentries, whereas for the larger atria the reentry was able to stabilize the arrhythmia during the entire simulation.

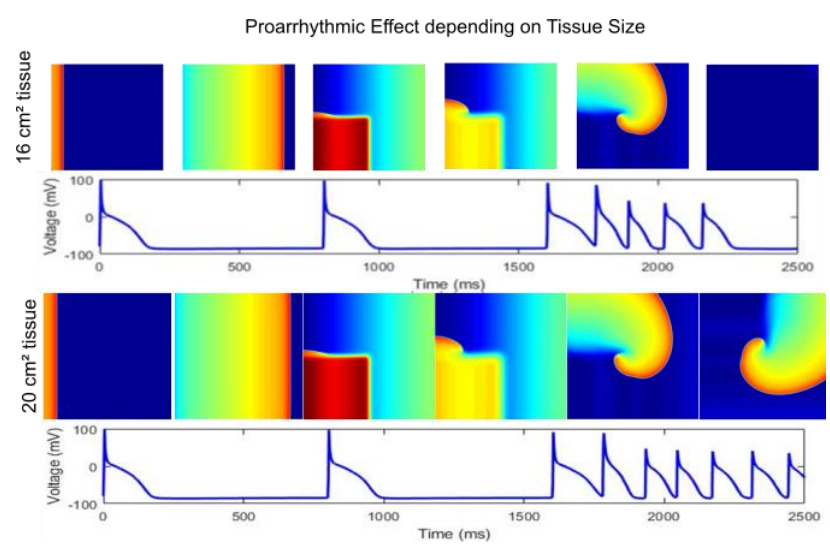

Figure 1. Example of proarrhythmic effect of a dilated atria.

Results for the entire database are shown in Table 1. As expected, larger atrium sizes allowed the maintenance of more reentries, and consequently the stabilization of $\mathrm{AF}$ in a larger number of models

Table 1. AF inducibility depending on atrial size.

\begin{tabular}{ccc}
\hline & $\begin{array}{c}\text { Basal } \\
\left(15 \mathrm{~cm}^{2}\right)\end{array}$ & $\begin{array}{c}\text { Dilated } \\
\left(20 \mathrm{~cm}^{2}\right)\end{array}$ \\
\hline $\begin{array}{c}\text { Mean AF maintenance } \\
\text { duration (ms) }\end{array}$ & $394 \mathrm{~ms}$ & $681 \mathrm{~ms}$ \\
\hline $\begin{array}{c}\text { Stable AF induced } \\
\left(\mathrm{n}^{\text {o }} \text { models }\right)\end{array}$ & $80 / 127$ & $88 / 127$ \\
\hline
\end{tabular}



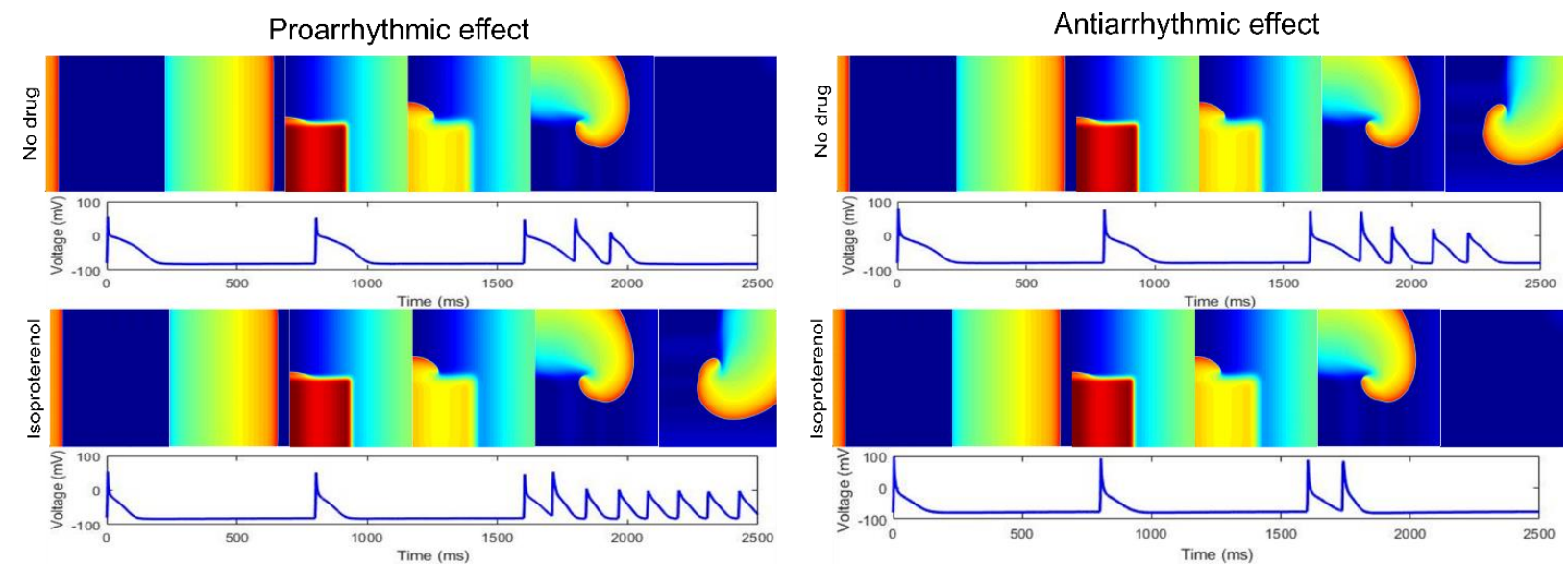

Figure 2. Example of proarrhythmic and antiarrhythmic effect of isoproterenol for two different models.

\subsection{Effect of drug on arrhythmogenicity}

Figure 2 illustrates opposed effects of the same drug concentration in two different models of the population for a size of $15 \mathrm{~cm}^{2}$. A proarrhythmic effect of isoproterenol can be observed in the left panel: whereas during basal conditions, the S2 pacing produced a reentry that selfterminated after a couple of reentries, after the administration of the isoproterenol, the S2 pacing produced a stable arrhythmia. Interestingly, in the example on the right isoproterenol produced an antiarrhythmic effect: during basal conditions, the $\mathrm{S} 2$ interval induced up to 4 reentries (i.e. $542 \mathrm{~ms}$ of reentry) but after the administration of the isoproterenol, the S2 pacing was only able to induce just one reentry (i.e. $111 \mathrm{~ms}$ of reentry).

Results for the entire database and both atrial sizes are depicted in Table 2. Interestingly, for basal size isoproterenol was proarrhythmic in 63 models $(49.32 \%)$, antiarrhythmic in 56 models (43.83\%) and had no effect in the remaining $8(6.85 \%)$.

For dilated size atria, isoproterenol was proarrhythmic in 81 models $(63.77 \%)$, antiarrhythmic in 42 models $(33.34 \%)$ and had no effect in the remaining $4(2.89 \%)$.

Table 2. Effect of isoproterenol on arrhythmogenicity

\begin{tabular}{ccc}
\hline Atrial size of $\mathbf{1 5} \mathbf{~ c m}^{\mathbf{2}}$ & Basal & Drug \\
\hline $\begin{array}{c}\text { Mean AF maintenance } \\
\text { duration (ms) }\end{array}$ & $717 \mathrm{~ms}$ & $1604 \mathrm{~ms}$ \\
$\begin{array}{c}\text { Stable AF induced } \\
\left(\mathrm{n}^{\mathbf{o}} \text { models }\right)\end{array}$ & $80 / 127$ & $84 / 127$ \\
\hline Atrial size of $\mathbf{2 0} \mathbf{~ c m}^{\mathbf{2}}$ & Basal & Drug \\
\hline $\begin{array}{c}\text { Mean AF maintenance } \\
\text { duration (ms) }\end{array}$ & $1313 \mathrm{~ms}$ & $1792 \mathrm{~ms}$ \\
$\begin{array}{c}\text { Stable AF induced } \\
\left(\mathrm{n}^{\text {o }} \text { models }\right)\end{array}$ & $88 / 127$ & $94 / 127$ \\
\hline
\end{tabular}

As observed, the same drug could be proarrhythmic or antiarrhythmic depending on the atrial size, highlighting that safety pharmacology should not be restricted to single cell simulations, but also at whole tissue level.

\subsection{Canonical Correlation Analysis (CCA)}

The analysis shown in sections 3.1 and section 3.2 required 525 hours of simulation in a nVIDIA TESLA C2057 GPU system. Extrapolation of those results to other atrial sizes, higher population of models of more drugs may become dramatically time consuming and unachievable for reasonable simulation periods.

Distribution of the 101 training sets of mathematical models over the CCA transformation are depicted in figure 3. As it can be observed, for both atrial sizes, the first component of the CCA model was able to differentiate between those models in which the isoproterenol was proarrhythmic (i.e. blue dots) and those in which the isoproterenol was antiarrhythmic (i.e. red dots). The second component (left panel) could serve to identify those models in which the drug had no effect (i.e. yellow dots).

Notice how the pattern of distribution of CCA components was similar for both atrial sizes confirming the robustness of the procedure.

When the trained regression model was used to estimate the effect of the drug on simulations not used for the training (20\%), a prediction with an accuracy of $96 \%$ for basal atria and $100 \%$ for dilated atria.

In addition to the predictability, CCA model allowed the identification of most relevant conductivities for the construction of the regression model. Specifically, for the basal atria of $15 \mathrm{~cm} 2$, the most influencing parameters were $\mathrm{gCaL}$, and $\mathrm{Nao}$, whereas for the dilated atria of $20 \mathrm{~cm} 2$, the most influencing parameters were $\mathrm{gNa}, \mathrm{gNak}$ and $\mathrm{gK} 1$. 

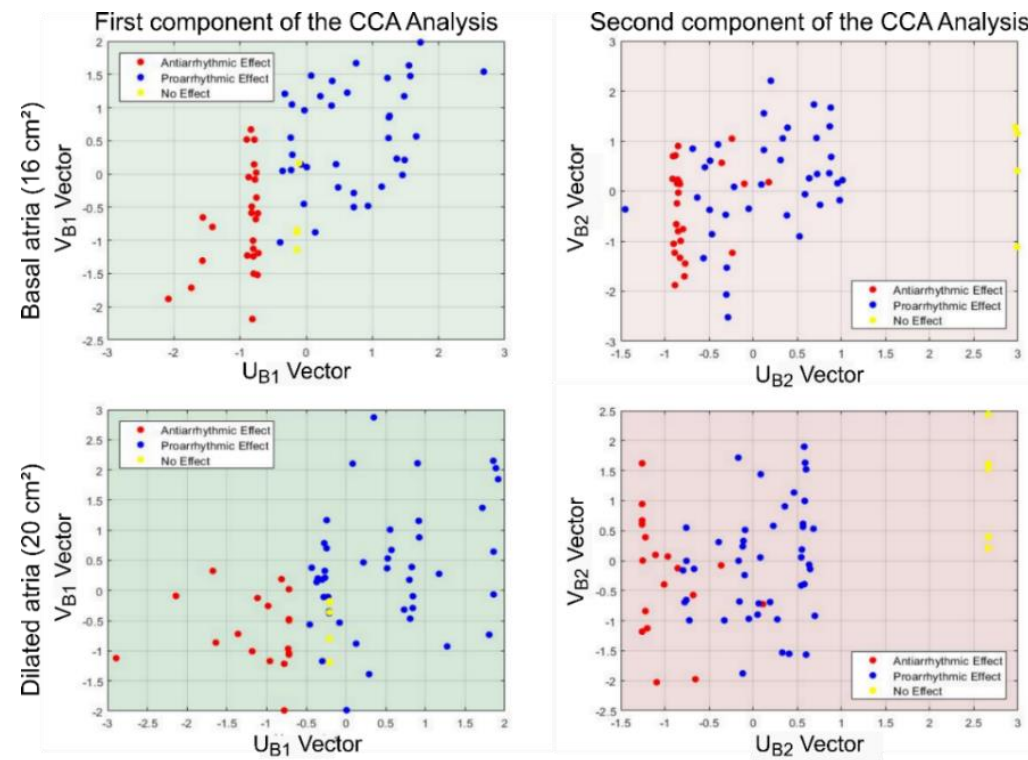

Figure 3. A. First Component of the CCA Analysis for small size atria B. Second Component of the CCA Analysis for small size atria C. First Component of the CCA Analysis for medium size atria D. Second Component of the CCA Analysis for medium size

\section{Discussion and conclusion}

A platform for the testing the pro and antiarrhytmicity of drugs in silico has been developed and evaluated on a set of 127 individuals in a multivariable scenario. The effect of drug on a population of models was studied for the case of isoproterenol in two different tissue sizes, accounting for either a healthy and dilated atria. This platform enables to evaluation of safety pharmacology considering realistic inter-subject variability.

In addition, a regression model based on CCA has demonstrated to be a robust procedure to predict the arrhythmogenicity of a drug without needing long and complex simulations. Furthermore, this methodology allowed the identification of most relevant parameters to predict drug effect.

Present results should be corroborated with a larger number of drugs and atrial tissue sizes, nevertheless, this approach provides a new opportunity to improve safety pharmacology tests and increase the efficacy of new drug developments, setting the base for personalized medicine.

\section{Acknowledgements}

This work was supported by the Instituto de Salud Carlos III FEDER (Fondo Europeo de Desarrollo Regional; IJCI- 2014-22178, DTS16/00160; PI16/01123; PI17/01059; PI17/01106)

\section{References}

[1] A. Liberos et al., "Balance between sodium and calcium currents underlying chronic atrial fibrillation termination: An in silico intersubject variability study," Hear. Rhythm, vol. 13, no. 12, pp. 2358-2365, Dec. 2016.

[2] A. Simon Chica et al., "Electrophysiological parameters in the electrical propagation during atrial fibrillation: a population of models study," Computing in Cardiology, vol. 44, pp. 1-4, 2017.

[3] R. Zou, J. Kneller, L. J. Leon, and S. Nattel, "Substrate size as a determinant of fibrillatory activity maintenance in a mathematical model of canine atrium," Am. $J$. Physiol. Circ. Physiol., vol. 289, no. 3, pp. H1002H1012, 2005.

[4] H. Oral et al., "Inducibility of paroxysmal atrial fibrillation by isoproterenol and its relation to the mode of onset of atrial fibrillation.," J. Cardiovasc. Electrophysiol., vol. 19, no. 5, pp. 466-470, May 2008.

[5] G. Vescovo, S. M. Jones, S. E. Harding, and P. A. PooleWilson, "Isoproterenol sensitivity of isolated cardiac myocytes from rats with monocrotaline-induced rightsided hypertrophy and heart failure.," J. Mol. Cell. Cardiol., vol. 21, no. 10, pp. 1047-1061, Oct. 1989.

Address for correspondence.

Name. Andreu M Ciment

Investigación Sanitaria Gregorio Marañón Hospital GU Gregorio Marañón. CIBERCV

Edificio Materno Infantil. c $\backslash$ O'Donnell 48, 28009, Madrid, Spain. E-mail address. acliment@ cibercv.es 